\title{
Giant aortic aneurysm in a patient with Marfan syndrome
}

\author{
Abstract \\ We report a case of clinically diagnosed Marfan syndrome complicated by severe \\ aortic regurgitation due to an aortic root aneurysm. The aneurysm had a maximal \\ diameter of $9.4 \mathrm{~cm}$. Despite this impressive dilatation of the aortic root, multimodality \\ imaging did not demonstrate aortic dissection.
}

Volume II Issue 5 - 2018

\author{
LH Joubert,' JT Janson, ${ }^{2}$ CG Kyriakakis' \\ 'Division of Cardiology, Department of Internal Medicine, \\ Stellenbosch University and Tygerberg Hospital, South Africa \\ ${ }^{2}$ Division of Cardiothoracic Surgery, Department of Surgery, \\ Stellenbosch University and Tygerberg Hospital, South Africa \\ Correspondence: LH Joubert, Division of Cardiology, \\ Department of Internal Medicine, Stellenbosch University and \\ Tygerberg Hospital, South Africa, Email Iloydjoubart@gmail.com
}

Received: August 17,2018| Published: October 09, 2018

\section{Introduction}

Marfan syndrome is an autosomal dominant multisystem connective tissue disorder due to a mutation in the gene encoding for fibrillin 1 (FBN 1). FBN 1 is not only a structural component of the extracellular matrix but also regulates transforming growth factor beta (TGF-B) expression. ${ }^{1}$ Abnormalities of TGF-B expression lead to the histological changes referred to as cystic medial necrosis, ${ }^{2}$ the underlying pathology of connective tissue leading to aortic dilatation in Marfan syndrome.

The major cause of morbidity and mortality in patients with Marfan syndrome is progressive aortic dilatation with its associated aortic dissection or rupture. ${ }^{3}$ Up to $80 \%$ of patients with Marfan syndrome will develop aortic dilatation during their lifetime. The risk of acute aortic syndromes increases as the aortic diameter increases. Patients with Marfan syndrome are considered to be at higher risk of dissection at lower aortic diameters. For this reason the absolute cut off for surgery in asymptomatic patients is $50 \mathrm{~mm}$ by The European Society of Cardiology (ESC) guidelines. ${ }^{4}$ A lower cut off of $45 \mathrm{~mm}$ is recommended in certain high-risk groups, including a family history of dissection and in females who are planning a pregnancy.

We present a case of a massively dilated aortic root in a patient fulfilling clinical criteria for the diagnosis of Marfan syndrome without aortic dissection.

\section{Case report}

A 29-year-old man was referred for inpatient assessment after he presented with a 2-month history of worsening orthopnoea with associated paroxysmal nocturnal dyspnoea. Despite these features of left heart failure, he did not give any history of effort intolerance. 1 week prior to presentation he presented to his general practitioner with a complaint of central chest pain which was intermittent and short lived, with no associated deterioration in his clinical condition. Of note in his previous surgical history, he underwent bilateral ophthalmic surgery for lens dislocation. Despite this, the diagnosis of Marfan syndrome was not considered at this time and no further screening was undertaken.
Clinical examination revealed an asymptomatic, mildly tachycardic patient at rest. His blood pressure was $140 / 50 \mathrm{mmHg}$ with a wide pulse pressure of $90 \mathrm{mmHg}$. A collapsing pulse could be palpated and Duroziez's sign could be elicited. The apex beat was displaced with a heaving quality and on auscultation first and second heart sounds were normal with a $3 / 6$ to-and-fro murmur audible. The diastolic component radiated down the right sternal border in keeping with aortic root dilatation as the cause for his AR.

Twelve lead ECG demonstrated a mild sinus tachycardia with an enlarged left atrium and the Sokolow-Lyon criteria for left ventricular hypertrophy were fulfilled.

A sitting AP chest radiograph demonstrated a significantly increased cardiac silhouette with a widened mediastinum (Figure 1). A CT aortogram demonstrated a severely dilated aortic root with aortic dilatation extending into the ascending aorta but normalising proximal to the brachiocephalic artery, sparing the aortic arch. (Figure 1)

His transthoracic echocardiogram (TTE) confirmed the presence of chronic severe aortic regurgitation with a severely dilated left ventricle and left atrium (Figure 2). Left ventricular systolic function was severely impaired with an ejection fraction (LVEF) of 30-35\%. Holodiastolic flow reversal was demonstrated in the descending thoracic aorta with diastolic flow reversal in the abdominal aorta (Figure 3). No primary valvular pathology was identified. The cause of the aortic regurgitation was severe dilation of the aortic root. On TTE the aortic root measured $8.4 \mathrm{~cm}$ at the sinuses of Valsalva (Figure 2). During intraoperative transoesophageal echocardiography (TOE) the maximum measurement at the sinuses was $9.4 \mathrm{~cm}$ (Figure 2). No evidence of aortic dissection was found on either of the 2 echocardiograms.

The patient was admitted to the cardiac ICU and underwent surgery the next day (Figure 4). A David's procedure (aortic root replacement with aortic valve reimplantation) was performed. Due to the extent of the aortic aneurysm, the distal anastomosis proximal to the brachiocephalic artery was performed under deep hypothermic arrest. The post-operative TOE showed a well-functioning aortic valve with minimal AR. 
Despite a recovery complicated by bleeding from the right coronary artery anastomosis, secondary to tension on the anastomotic suture site, requiring relook sternotomy, our patient was successfully discharged 19 days after surgery. He was reviewed in our clinic 1 year post procedure and he is well with no further heart failure symptoms. His LVEF has unfortunately not recovered despite appropriate medical therapy.
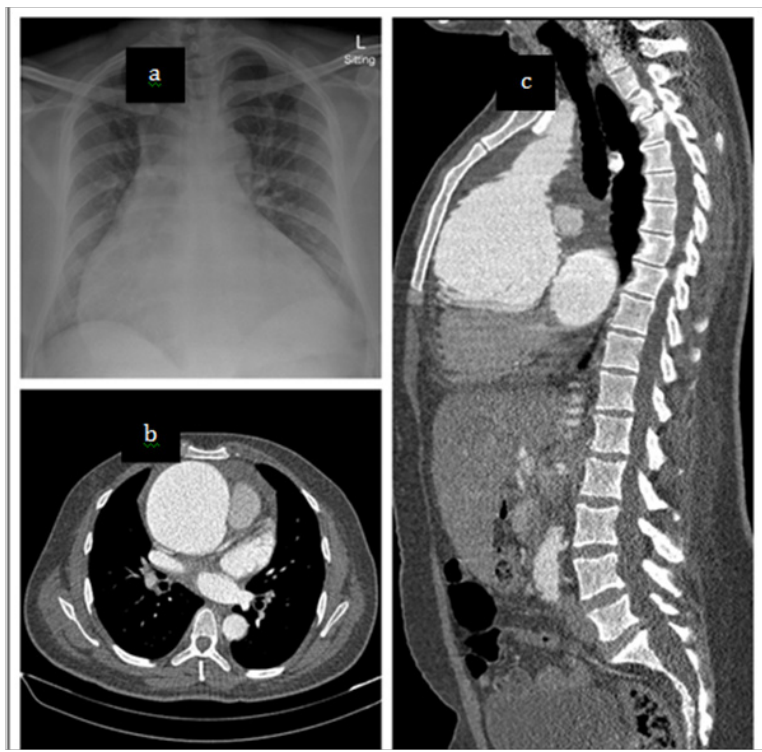

Figure I Chest radiograph and CT aortogram. a) sitting AP chest x-ray demonstrating enlarged cardiac silhouette and widened mediastinum. b) CT scan at aortic valve level demonstrates the absence of dissection with aneurysm measuring $99.5 \mathrm{~mm}$ at its widest diameter. c) Sagittal plane reconstruction demonstrating the extent of the aneurysm.
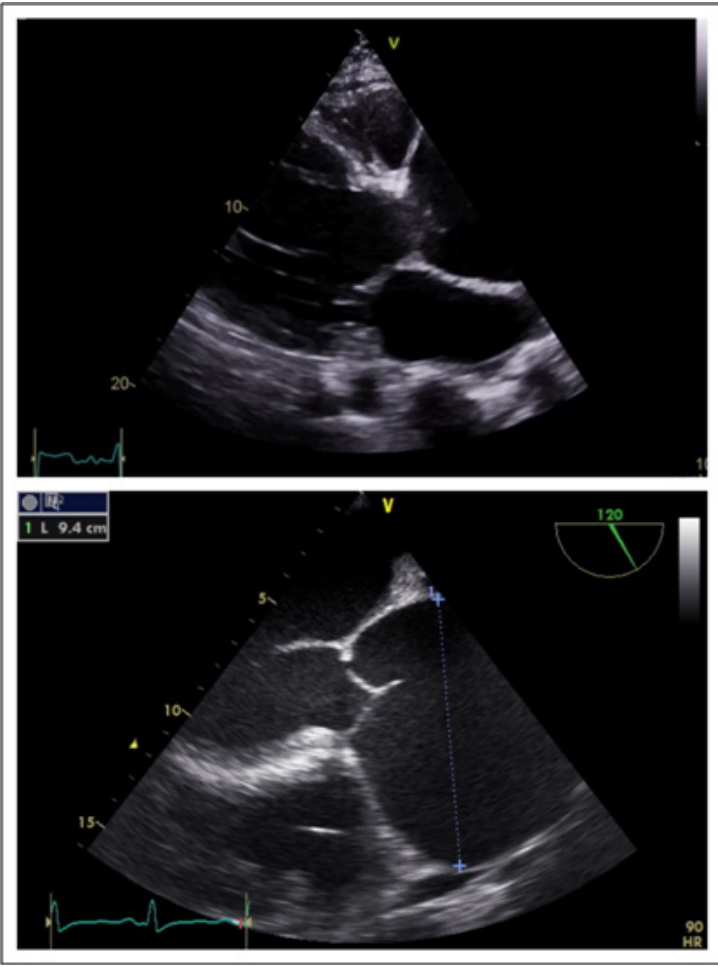

Figure 2 Dilated aortic root a) Trans thoracic echocardiogram parasternal long axis demonstrating aortic root dilatation initially estimated to be $8.3 \mathrm{~cm}$ b) Transoesophageal echocardiogram mid oesophageal view at 120 degrees long axis view demonstrating maximal diameter of aortic root to be $94 \mathrm{~mm}$.

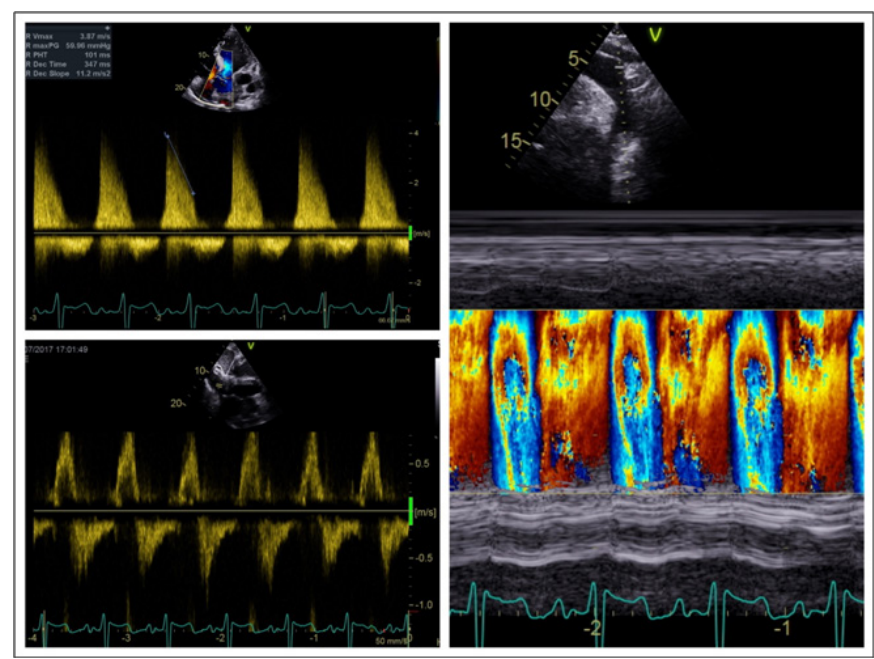

Figure 3 Severe aortic regurgitation. a) trans aortic valve continuous wave Doppler demonstrating severe aortic regurgitation with pressure half time of $10 \mathrm{Ims}$. b) Pulse wave Doppler in the abdominal aorta demonstrating diastolic flow reversal. c) Colour M-mode in the descending aorta demonstrating holodiastolic flow reversal.

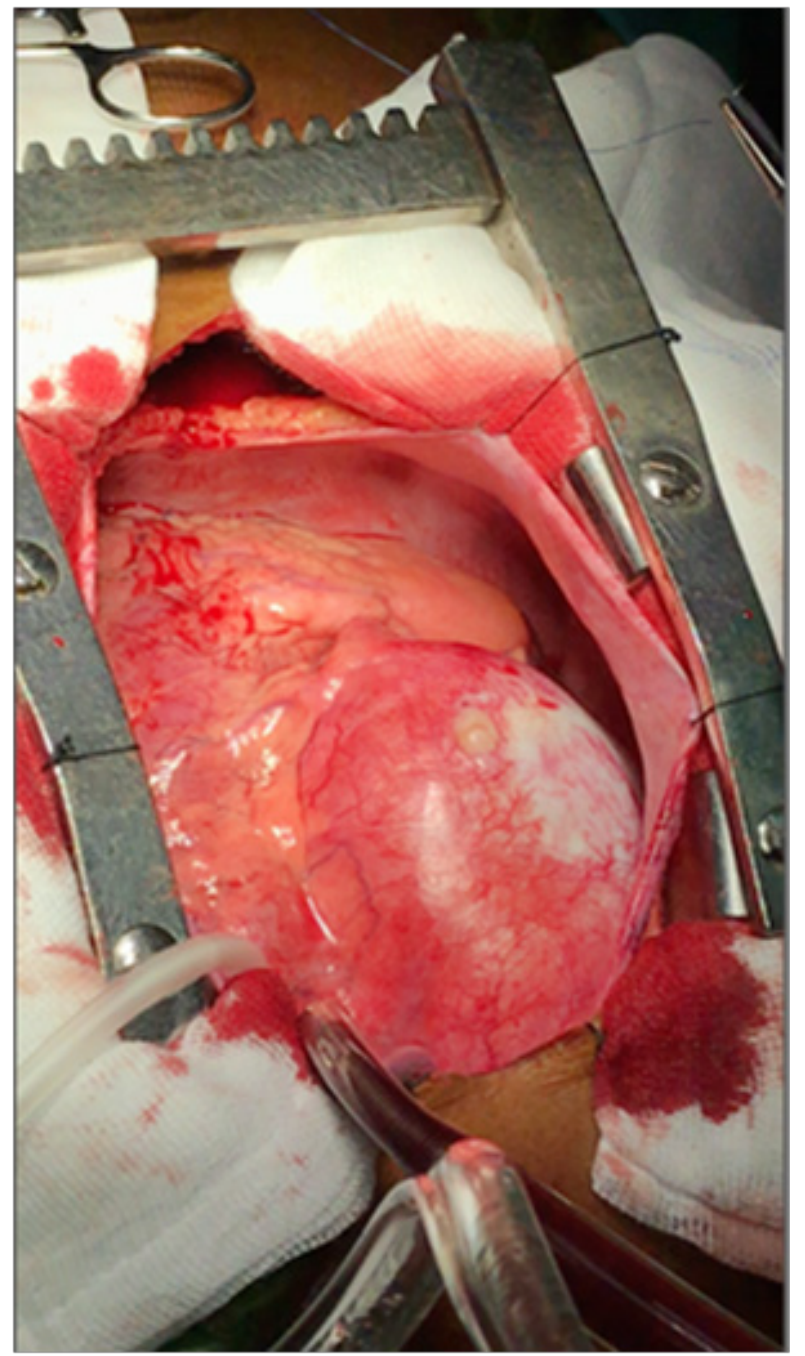

Figure 4 Operative video. Operative video taken from the head of the bed. Midline sternotomy with pericardium open. Massively dilated aorta can be seen cephalad to the heart. 


\section{Discussion}

The diagnostic criteria for Marfan syndrome were revised in $2009 .{ }^{5}$ In the revised Ghent nosology more emphasis is placed on positive genetic testing for FBN1, but avoids making this a mandatory requirement for diagnosis. Ectopia lentis and aortic root dilatation, the most common clinical manifestations of the disease, remain sufficient criteria for establishing a definitive diagnosis of Marfan syndrome. For this reason, it would seem prudent that any patient presenting with ectopia lentis or lens dislocation should be screened for Marfan syndrome. This enables the clinician not only to diagnose and screen for a potentially lethal condition and its complications, but also to institute prophylactic therapy that can modify the disease course. ${ }^{3,4}$

There have been many studies published in the literature attempting to elucidate the best medical therapy for the prevention of progressive aortic dilatation and its attendant risk of dissection. In a recent review of drug trials in Marfan syndrome it was summarised that angiotensin receptor antagonists (ARBs) are as effective as beta blockers (BBs) at reducing the rate of progressive aortic dilatation. Not only did the Paediatric Heart Network trial ${ }^{7}$ confirm ARBs and BBs are both equally effective, it also demonstrated that younger patients were more likely to respond to therapy. This would suggest that early treatment may be more effective.

While both monotherapies appear to be equally effective, there is conflicting evidence in terms of combination ARB and BB therapy. The MARFAN SARTAN ${ }^{8}$ trial demonstrated no benefit to adding losartan to BB therapy. While COMPARE ${ }^{9}$ showed that the addition of losartan to standard therapy reduced the rate of progressive aortic dilatation, there was no difference in mortality or aortic dissection outcomes. Again in the paediatric and adolescent population, the response to combination therapy appears to be slightly better than with either drug alone seeing mildly decreased aortic dilatation over 35 -months of follow up. ${ }^{10}$

These findings are reflected in current clinical practice. The current ESC guideline ${ }^{3}$ recommends beta blockade for all patients "... to prevent aortic complications..." but does not differentiate between those with and without dilated aortas. In their review of recent drug trials in Marfan syndrome, Singh et al. ${ }^{6}$ recommend using a $\mathrm{Z}$ score for aortic dilatation of more than 2.5 as a threshold for the initiation of medical therapy. In those with a $\mathrm{Z}$ score of less than 2.5 it is reasonable to practice watchful waiting and initiate therapy should aortic dilatation occur. Patients with high risk features such as a family history of aortic aneurysm or dissection, increased aortic stiffness and increased vertebral artery tortuosity should be placed on medical therapy regardless of $\mathrm{Z}$ score. The authors further recommend that combination therapy be reserved for patients with severe, progressive aortic dilatation ( $\mathrm{Z}$ score above 5$)$

\section{Conclusion}

While Marfan syndrome has a variable phenotype with multiple systemic features, the modified Ghent Criteria make the diagnosis relatively simple. In patients diagnosed with Marfan syndrome it is imperative to institute surveillance measures to assess the aortic root as complications of aortic root dilation are the major cause of morbidity and mortality. Once present it is appropriate to institute medical therapy in the form of either a beta blocker or angiotensin receptor antagonist to slow progression of aortic dilatation. Prophylactic surgery at prespecified guideline driven cut-offs, affords the clinician the opportunity to intervene in high risk individuals while still in a stable asymptomatic condition.

\section{Acknowledgements}

None.

\section{Conflict of interest}

The authors declare that there is no conflict of interest.

\section{References}

1. Habashi JP, Judge DP, Holm TM, et al. Losartan, an AT1 antagonist, prevents aortic aneurysm in a mouse model of Marfan syndrome. Science. 2006;312(5770):117-121.

2. Dietz H. Marfan Syndrome. In: Adam MP, Ardinger HH, et al., editors. Seattle (WA), USA, 1993

3. Baumgartner H, Bonhoeffer P, De Groot NMS, et al. ESC Guidelines for the management of grown-up congenital heart disease (new version 2010). Eur Heart J. 2010;31(23):2915-2957.

4. Erbel R, Aboyans V, Boileau C, et al. 2014 ESC guidelines on the diagnosis and treatment of aortic diseases. Eur Heart J. 2014;35(41):2873-2926.

5. Loeys BL, Dietz HC, Braverman AC, et al. The revised Ghent nosology for the Marfan syndrome. J Med Genet. 2010;47(7):476-485.

6. Singh MN, Lacro R V. Recent Clinical Drug Trials Evidence in Marfan Syndrome and Clinical Implications. Can J Cardiol. 2016;32(1):66-77.

7. Linte CA, Camp JJ, Rettmann ME, et al. Atenolol versus losartan in children and young adults with Marfan's syndrome. NEJM. 2014;371(22):2061-2071

8. Milleron O, Arnoult F, Ropers J, et al. Marfan Sartan: a randomized double-blind, placebo-controlled trial. Eur Heart J. 2015;36(32):2160 2166 .

9. Groenink M, Den Hartog AW, Franken R, et al. Losartan reduces aortic dilatation rate in adults with Marfan syndrome: A randomized controlled trial. Eur Heart J. 2013;34(45):3491-3500.

10. Chiu H-H, Wu M-H, Wang J-K, et al. Losartan added to beta-blockade therapy for aortic root dilation in Marfan syndrome: a randomized, openlabel pilot study. Mayo Clin Proc. 2013;88(3):271-276. 